\title{
Organelle asymmetry for proper fitness, function, and fate
}

\author{
Dorothy A. Lerit • Jeremy T. Smyth • \\ Nasser M. Rusan
}

(C) Springer Science+Business Media Dordrecht (Outside the USA) 2013

\begin{abstract}
During cellular division, centrosomes are tasked with building the bipolar mitotic spindle, which partitions the cellular contents into two daughter cells. While every cell will receive an equal complement of chromosomes, not every organelle is symmetrically passaged to the two progeny in many cell types. In this review, we highlight the conservation of nonrandom centrosome segregation in asymmetrically dividing stem cells, and we discuss how the asymmetric function of centrosomes could mediate nonrandom segregation of organelles and mRNA. We propose that such a mechanism is critical for insuring proper cell fitness, function, and fate.
\end{abstract}

Keywords Centrosome - Asymmetric · Organelle · Mitosis $\cdot$ Stem cell

$\begin{array}{ll}\text { Abbreviations } \\ \text { Cnb } & \text { Centrobin } \\ \text { D } & \text { Daughter (centriole) } \\ \text { ER } & \text { Endoplasmic reticulum } \\ \text { GD1 } & \text { Granddaughter (centriole) 1 } \\ \text { GD2 } & \text { Granddaughter (centriole) 2 }\end{array}$

Responsible Editor: Helder Maiato and Yves Barral

D. A. Lerit $\cdot$ J. T. Smyth $\cdot$ N. M. Rusan $(\bowtie)$

Cell Biology and Physiology Center, National Heart, Lung, and Blood Institute, National Institutes of Health, Bethesda, MD, USA

e-mail: Nasser@nih.gov

$\begin{array}{ll}\text { M } & \text { Mother (centriole) } \\ \text { mGSCs } & \text { Male germline stem cell } \\ \text { MT } & \text { Microtubule } \\ \text { MTOC } & \text { Microtubule organizing center } \\ \text { NB } & \text { Neuroblast } \\ \text { PCM } & \text { Pericentriolar material } \\ \text { RGC } & \text { Radial glial cells } \\ \text { SOP } & \text { Sensory organ precursor } \\ \text { SPB } & \text { Spindle pole body }\end{array}$

\section{Introduction}

As the microtubule (MT)-organizing centers (MTOCs) of eukaryotic cells, centrosomes function in diverse cellular processes. Centrosomes are perhaps best known for their role in organizing the bipolar mitotic spindle, which cleaves a parent cell into two daughter cells during cellular division. However, centrosomes perform critical tasks in non-mitotic cells as well, such as polarizing cell growth, directing cell migration, forming specialized cellular organelles called cilia and flagella, and guiding the intracellular trafficking of various cargoes. In this review, we discuss the critical role of centrosomes in establishing cellular asymmetry in interphase with an emphasis on how centrosomes contribute to the nonrandom segregation of organelles and mRNA in dividing stem cells.

Centrosomes are complex, multi-layered organelles composed of centrioles, a possible transition zone, and 
an overlying matrix of pericentriolar material, or PCM. Each centrosome contains an inner core of two centrioles, often described as barrel-like structures, which are oriented orthogonally to each other (Fig. 1a). Centrioles share a basic structure comprising a stereotypical arrangement of nine MT triplets; however, the two centrioles of a given centrosome are not equivalentone centriole is always older than the other. In G1 of the cell cycle, the single centrosome contains a mother (M) and a daughter (D) centriole, which differ in age, ultrastructure, and protein composition (discussed in detail below). Disengagement of these two centrioles allows each the ability to template a granddaughter centriole (GD1 and GD2). The result is a "mother centrosome" that contains the M-GD1 centriole pair and a "daughter centrosome," which contains the D-GD2 centriole pair (Fig. 1a). Therefore, in a cell containing two centrosomes it is always the case that one is older or more mature (Fig. 1b). This intrinsic biological clock that distinguishes the two centrosomes creates the perfect mechanism to exploit during the segregation of two populations of nucleic acids, proteins, or organelles within a single cell. This mechanism might be particularly important in stem cell asymmetric division, where distinguishing the two daughter cells after mitosis is critical.

In contrast to the well-defined and relatively static structure of centrioles, PCM has often been described as a dynamic, amorphous cloud. The activity, or MT nucleation capacity, of a centrosome directly relates to the amount of PCM surrounding the centrioles. Centrosome activity fluctuates throughout the cell cycle, peaking in mitosis when both PCM levels and MTOC capacity are highest (Khodjakov and Rieder 1999; Sunkel et al. 1995). Live-imaging studies show that these fluctuations in PCM levels are controlled independently on the mother and daughter centrosome in Drosophila stem cells (Conduit et al. 2010; Rebollo et al. 2007; Rusan and Peifer 2007), indicating that a highly complex mechanism can locally (at the centrosome) control maturation and activity. Recent studies using structured-illumination microscopy have upended the traditional description of PCM as being amorphous (Fu and Glover 2012; Lawo et al. 2012; Mennella et al. 2012). It will be exciting to test how this new, more structured view of PCM relates to function in vivo, especially in stem cells where the mother and daughter centrosomes are clearly functionally different.

This review focuses on how and why different stem cells regulate the inheritance of the mother and daughter centrosomes. We also discuss the presence of one active and one inactive centrosome in interphase, a common feature of Drosophila stem cells. The role of this one active interphase centrosome in cortical interaction, polarity establishment, segregation of cell fate determinants, spindle alignment, and asymmetric
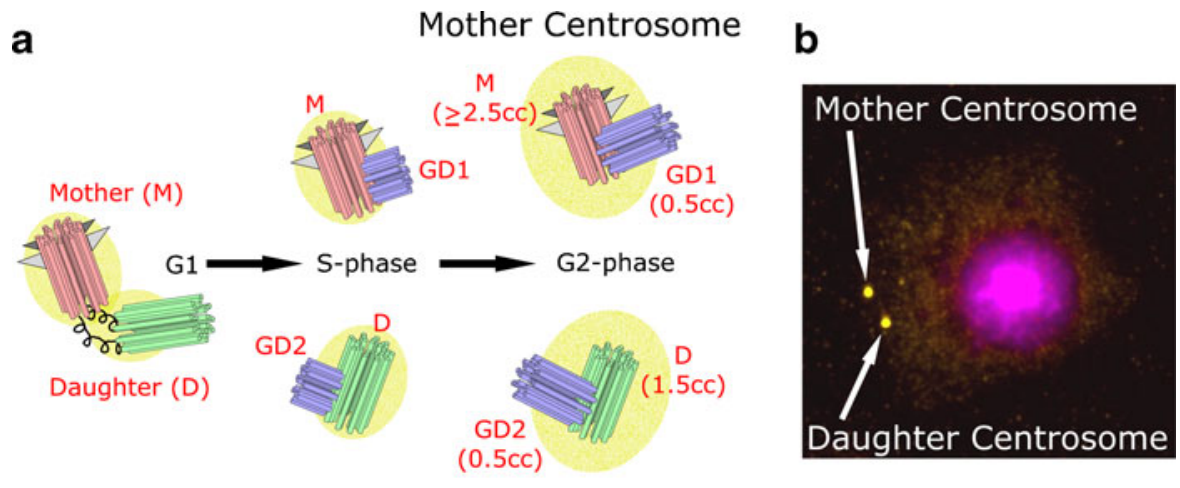

\section{Daughter Centrosome}

Fig. 1 Model of centrosome duplication. Centrosome duplication occurs in S-phase resulting in two centrosomes that are capable of organizing PCM in G2 (Callaini and Riparbelli 1990). a A cartoon representation of mammalian centriole duplication and mother-daughter centrosome formation. In early interphase, a cell receives a single centrosome containing a mother $(M$, with distal and subdistal appendages (Vorobjev and Chentsov 1980) and a daughter $(D)$ centriole pair. In S-phase, the $\mathrm{M}-\mathrm{D}$ disengaged centrioles each nucleate a granddaughter (GD) centriole to form a M-GD1 pair (mother centrosome) and a D-GD2 pair (daughter centrosome). The age of each centriole can be roughly calculated in units of cell cycles (cc). b Cultured Drosophila S2 cell in G2 stained for DNA (pink) and centrosomes (yellow). Mother-daughter identity is not actually known; the labeling is meant for illustration 
division has been discussed in several reviews (Lesage et al. 2010; Yamashita and Fuller 2008). Here, we discuss the possible significance of this asymmetric centrosome activity as it relates to asymmetric organelle and mRNA segregation.

Choosing a mother's wisdom or a daughter's youth

During cell division, each progeny cell will receive either the mother centrosome or the daughter centrosome. In symmetrically dividing cells, the distinction between which cell acquires the mother versus the daughter centrosome is rarely considered. However, even in symmetrically dividing cultured cells, asymmetric centrosome inheritance determines which one of the two progeny cells is first to build its primary cilium (Anderson and Stearns 2009). This discrepancy may have a significant effect on the signaling capacity of individual cells. Investigating these differences in symmetrically dividing cells in a vertebrate animal model will be an exciting future direction. Moreover, biased segregation of the two centrosomes could have profound cell fate significance, especially during asymmetric cell division. Therefore, it is critical to understand the patterns of centrosome segregation in multiple systems in order to identify both unique and common mechanisms.

Budding yeast A stereotypical pattern of motherdaughter centrosome inheritance was first described in the budding yeast, Saccharomyces cerevisiae. Frequently compared to stem cell divisions, budding yeast undergo repeated rounds of asymmetric cell division, with a smaller daughter cell budding off from the larger mother cell (Fig. 2a). The yeast MTOC is called the spindle pole body (SPB), and it is analogous to the centrosome of higher eukaryotes. With every yeast mitotic division, one SPB segregates to the bud and another SPB is retained within the mother cell.

The observation of the asymmetric association of certain proteins with the bud SPB motivated the investigation of biased segregation of centrosomes. Early structure-function and genetic experiments suggested strict SPB inheritance patterns (Vallen et al. 1992). Later live-cell-imaging experiments conclusively demonstrated that the mother SPB is inherited by the bud cell (Pereira et al. 2001). However, disrupting the inheritance pattern using MT depolymerization-repolymerization experiments did not uncover the significance of this inheritance pattern (Pereira et al. 2001).

Drosophila germline stem cells Biased centrosome inheritance was subsequently demonstrated in the Drosophila male germline stem cells (mGSCs). mGSCs are arranged around a supportive niche called the hub and stereotypically divide along an apicalbasal axis to effectively displace the differentiating cell, or gonialblast, away from the hub (Yamashita et al. 2003). This oriented asymmetric division ensures the mGSC, and not the gonialblast, remains in close proximity to the stem cell niche (Fig. 2b). Genetic studies strongly implicate the physical association of one centrosome with the mGSC-hub interface in the fixed orientation of the mGSC mitotic spindle (Inaba et al. 2010; Yamashita et al. 2003). Subsequent work revealed the mother centrosome associates with the mGSC-hub cortex and is retained within the stem cell, while the daughter centrosome is passaged to the gonialblast (Yamashita et al. 2007). The distinct centrosome inheritance patterns observed in yeast versus mGSCs suggest that modes of centrosome inheritance are largely cell-type specific.

Nonetheless, the biased segregation of centrosomes into two differentially fated cells led to the speculation that perhaps centrosomes, or their asymmetrically localized cargoes, autonomously guide cell-fate decisions. In fact, in the case of both mGSCs and budding yeast division, the mother centrosome partitions to the progeny cell that has the greatest proliferative capacity. mGSCs continually divide throughout adult life, whereas gonialblasts have a limited replicative potential and divide exactly four times during spermatogenesis (Fuller 1993). Likewise, the yeast mother SPB is passaged into the bud, which has a greater reproductive potential than the yeast mother cell (Henderson and Gottschling 2008). Dubbed the "immortal centrosome hypothesis" (Morrison and Spradling 2008), this relationship between the mother centrosome and proliferative potential has been challenged by studies of the neural stem cells, or neuroblasts (NBs), of the Drosophila larval central nervous system.

Drosophila neural stem cells Similar to mGSCs, NBs asymmetrically divide along an invariant apical-basal axis and generate unequally fated progeny cells by retaining a single centrosome at the apical cortex throughout the cell cycle (Januschke and Gonzalez 
a

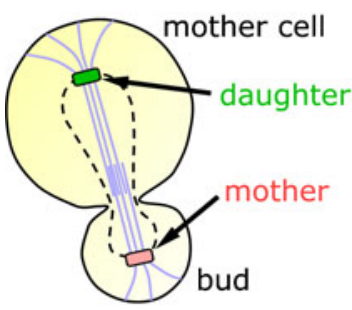

S. cerevisiae b

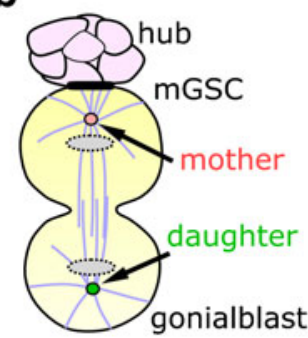

Drosophila mGSCs

Fig. 2 Centrosome inheritance patterns. The inheritance of the mother or daughter centrosome is highly dependent on cell type. Shown are four examples of this selective inheritance: a budding yeast, S. cerevisiae (Pereira et al. 2001), b Drosophila male germline

2010; Rebollo et al. 2007; Rusan and Peifer 2007). With each mitotic division, NBs self-renew and give rise to a much smaller daughter cell, called a ganglion mother cell (GMC), such that each stem cell is associated with a cluster of GMCs (Fig. 2c). Unlike the highly proliferative NBs, GMCs generally only divide once or twice and then differentiate to form neuronal cells (Homem and Knoblich 2012).

Based on the different proliferative capacities of these cell types, the immortal centrosome hypothesis would predict that the mother centrosome is specifically retained by the NBs. However, this is not the case. The first indication that NBs retain the daughter centrosome came from the observation of unequal centriole labeling (using GFP-PACT) of the mother and daughter centrosomes (Conduit and Raff 2010). In parallel studies, the same NB pattern of centrosome inheritance was elegantly demonstrated by the identification of a daughter-centrosome specific protein, Centrobin $(\mathrm{Cnb})$, and by tracking photoconvertible centrioles over multiple cell cycles (Januschke et al. 2011). Importantly, the findings in Drosophila NBs and mGSCs indicate that even within a single model organism, the inheritance pattern of centrosomes is cell-type specific. As is the case with budding yeast, the significance of the inheritance pattern in both mGSCs and NBs has not been determined.

Mouse neural stem cells Remarkably, the link between asymmetric cell division and nonrandom centrosome inheritance is conserved in neural stem cells of both mice and humans. The first of the mammalian cell
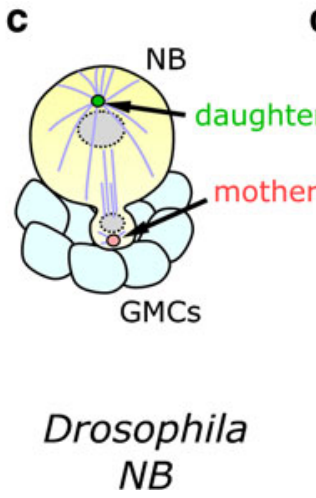

d

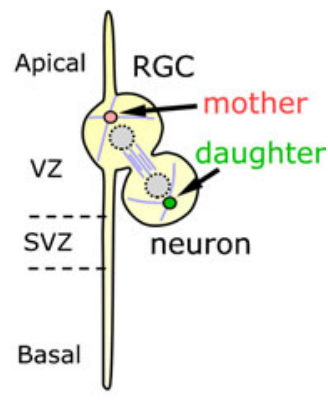

Mouse NB stem cells (mGSCs; Yamashita et al. 2007), c Drosophila larval neural stem cells (NB; Conduit and Raff 2010; Januschke et al. 2011), and d mouse radial glia cells (RGC; Wang et al. 2009)

studies showed a distinct centrosome segregation pattern in mouse radial glia cells (RGCs). RGCs are neuronal precursor cells of the mammalian neocortex that divide asymmetrically during the peak phase of neurogenesis (Fig. 2d). By cotransfecting mice with both RFP-Centrin, which labels all centrioles, and GFP-Ninein, a mother-centrosome specific marker, it was shown that RGCs specifically retain the mother centrosome. The segregation pattern of the RGC centrosomes was then definitively demonstrated by impressive live cell imaging of photoconvertible centrioles (Wang et al. 2009). The researchers attempted to test the significance of this inheritance by knocking down the mother centriole protein Ninein; the result is a massive loss of RGCs (Wang et al. 2009). Unfortunately, these data do not prove that randomization of centrosome segregation is responsible for stem cell loss. Loss of Ninein does not necessary mean loss of mother centrosome identity; a secondary role of Ninein might be at play. Interestingly, however, this observation that RGCs retain the mother centrosome, while Drosophila NBs retain the daughter centrosome, indicates that centrosome segregation patterns are not conserved among all neuronal stem cells.

Cultured human cancer cells The most recent study of asymmetric centrosome segregation was performed in human neuroblastoma cell lines. Neuroblastomas are solid brain tumors that arise from mutations in neuronal precursors, called neural crest cells (reviewed in Jiang et al. 2011). The authors showed that cells from these cell lines are polarized and asymmetrically 
segregate their centrosomes (Izumi and Kaneko 2012). These studies highlight another correlation between asymmetric centrosome inheritance and asymmetric division. However, it is still unknown if cell polarization, or cell fate, relies on mother-daughter centrosome asymmetry. This culture system should be investigated further, possibly using mouse neuroblastoma cell lines to allow the comparison to normal mouse neural crest cells.

In summary, it is still unclear why disparate systems differentially retain daughter or mother centrosomes, although it is tempting to speculate why one centrosome may confer a certain advantage over another. For example, it has been proposed that the passage of the mother centrosome to GMCs correlates with the long lifespan of the neurons formed by these cells (Januschke et al. 2011), but this explanation does not hold true for mouse RGCs. Another hypothesis is that a daughter centrosome is retained in NBs to avoid any damage that may accumulate on an older centrosome. Simply put, the physiological significance of preferential centrosome segregation is unknown and is likely to vary from one stem cell type to the next.

The bigger they are the harder they haul

Regardless of which centrosome is inherited, budding yeast, Drosophila mGSCs, Drosophila NBs, and possibly mouse RGCs all share a common characteristicat some point during interphase, one centrosome is more active as an MTOC than the other (Fig. 3). In budding yeast, there is a 10-min delay between the full maturation of the two SPBs (Shaw et al. 1997). In NBs, the daughter centrosome is active and the mother is inactive throughout interphase (Rebollo et al. 2007; Rusan and Peifer 2007). In mGSCs, the mother centrosome organizes a microtubule aster that is larger than the daughter centrosome in interphase (Yamashita et al. 2007). Finally, we might speculate that the mother centrosomes in RGCs could anchor more microtubules, but this has not been shown (Wang et al. 2009). Why might a cell want an imbalance in MTOC activity in interphase, even for a short period of time? For a discussion on the role of this active MTOC in asymmetric division, please refer to the following reviews (Lesage et al. 2010; Yamashita and Fuller 2008). We would like to discuss the possible role of the MTOC in asymmetric organelle and mRNA segregation. Given the lack of information from any stem cell model system, we use data from yeast, mammalian culture cells, and Drosophila for our speculation and hypotheses.

\section{Biased segregation of mitochondria and endoplasmic reticulum}

Properly partitioning organelles to progeny cells is an important aspect of cell proliferation. Several organelles, including mitochondria and the endoplasmic reticulum (ER), cannot be formed de novo; therefore, daughter cells need to inherit a critical amount of starting material during cell division. However, it may not simply be important for cells to acquire a threshold quantity of organelles; it may also be important that they acquire a threshold quality of organelles. This may be especially true in asymmetric stem cell divisions, in which delineation of cell fate and function is critically important. Organelle quality may be related to organelle age and could be a measure of functional capacity. This concept of functional segregation of organelles is therefore similar to the already described age-based selection of centrosomes.

Segregation of organelles other than centrosomes has not been analyzed in dividing stem cells. However, division of budding yeast offers examples of selective organelle segregation during asymmetric cell division. It has been shown that mitochondria in budding yeast, for example, are partitioned into two unequal pools at each mitotic division, such that the daughter cell might exclusively acquire the most functional, metabolically active mitochondria (Fig. 4a). Partitioning of mitochondria to the mother cell versus the bud depends on differential interactions of mitochondria with F-actin cables that extend through the bud neck. The actin cables themselves undergo retrograde movement from the bud to the mother cell (Yang and Pon 2002), and association of mitochondria with $\mathrm{F}$-actin results in transport of mitochondria toward the distal pole of the mother cell. However, a fraction of mitochondria also exhibit anterograde transport along the F-actin cables into the bud (Fehrenbacher et al. 2004; Simon et al. 1997). The retrograde movement of mitochondria to the distal mother pole can be viewed as a passive process, whereby mitochondria simply hitch a ride on the retrograde moving actin cables. However, the anterograde transport to the bud requires that the mitochondria move along F-actin cables that are themselves moving in the opposite direction. Thus, 


\section{a Interphase MTOC asymmetry}

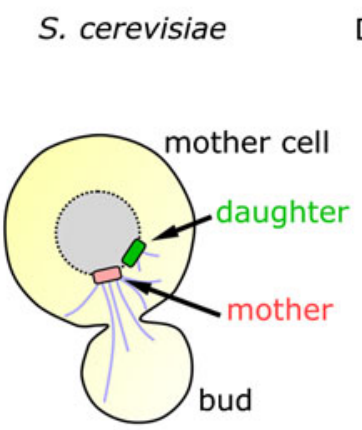

Drosophila mGSCs

Drosophila NB
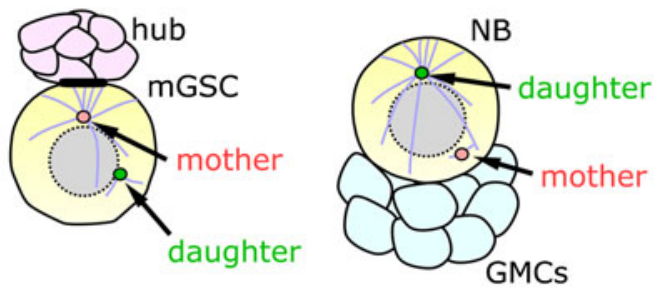

Mouse NB

(hypothetical)

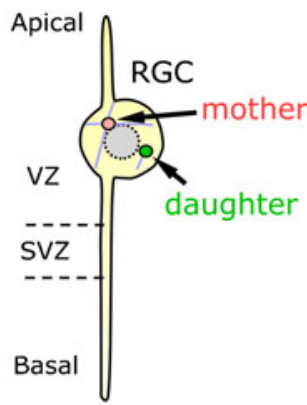

\section{b Mechanism of interphase MTOC asymmetry}

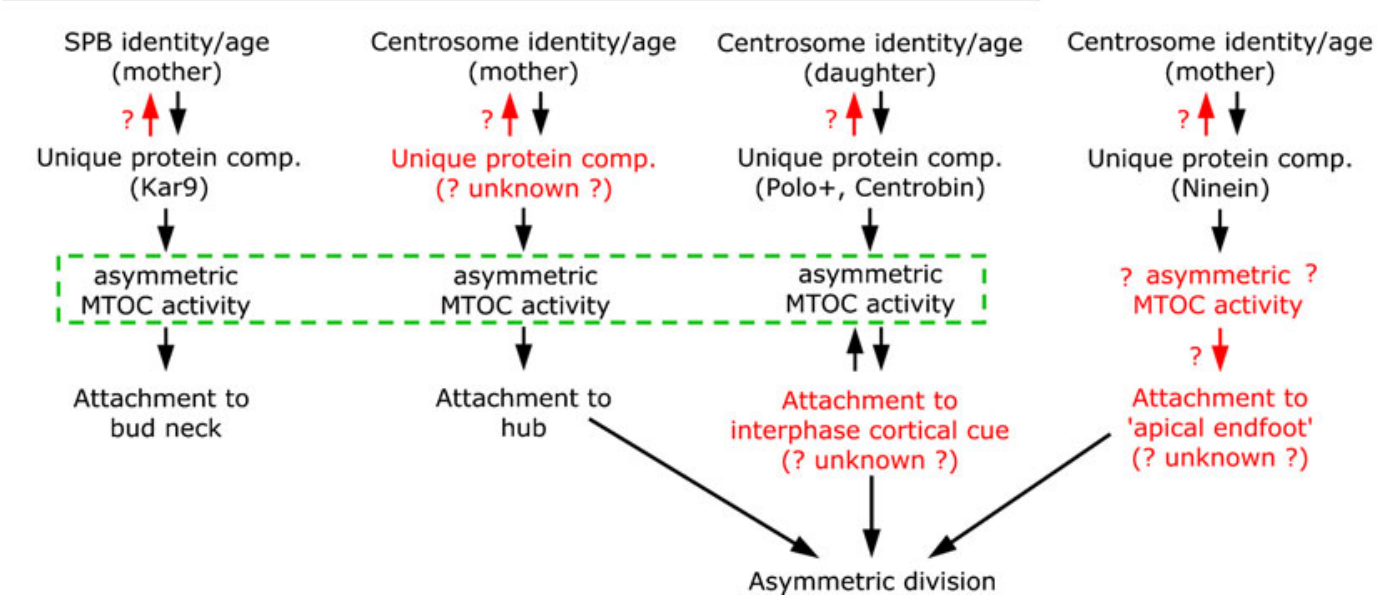

Fig. 3 Interphase MTOC asymmetry. a A cartoon illustrating a single interphase MTOC in each of four cell types. Supportive data does exist for budding yeast (Shaw et al. 1997), Drosophila male germline stem cells (mGSCs; Yamashita et al. 2007), and Drosophila larval neural stem cells (NBs; Januschke and Gonzalez 2010; Rebollo et al. 2007; Rusan and Peifer 2007). The mouse RGCs are not known to use such a mechanism. b Shows a summary of what little we know of the process of establishing MTOC asymmetry in interphase. The role of Kar9 in SPBs (Hotz et al. 2012; Liakopoulos et al. 2003; Miller and Rose 1998) and Centrobin in NBs (Januschke et al. 2013) are the best-characterized mechanisms thus far. Red text indicates all the hypotheses that need further investigation

juxtaposed with the nucleus, is referred to as perinuclear ER. A second domain of the yeast ER, known as cortical ER, lies just beneath the plasma membrane. These two ER domains are connected by thin ER tubules that traverse the cytoplasm. During division, the perinuclear ER is evenly distributed between the mother and bud cells by the mitotic spindle concomitant with nuclear division. In contrast, a fraction of the cortical ER is specifically transported into the growing bud along F-actin cables by the myosin V motor, Myo4p (Du et al. 2004; Lowe and Barr 2007). Thus, it is already evident that different domains of the ER are differentially partitioned to the 


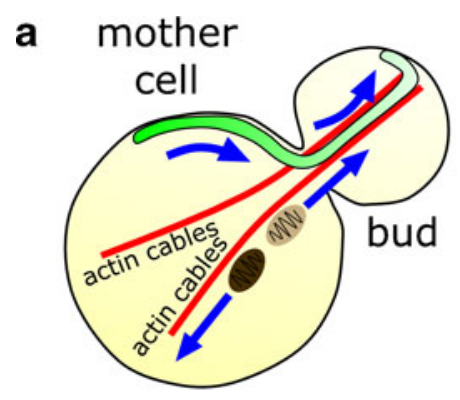

C

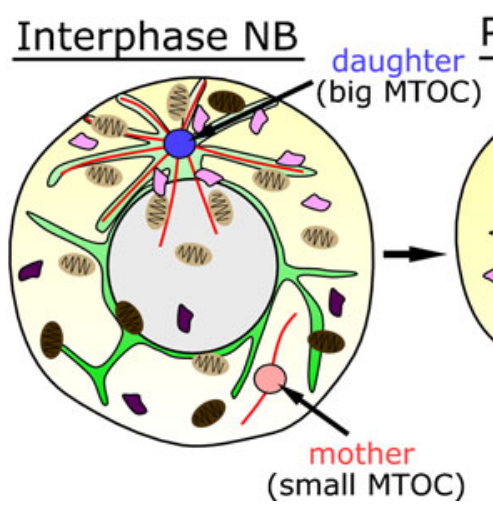

Fig. 4 Asymmetric organelle inheritance. a Data from yeast suggest that both ER and mitochondria are selectively segregated into the mother cell or bud (Du et al. 2004; Lowe and Barr 2007; Peraza-Reyes et al. 2010). b A mammalian cell showing the sequestration of an aggresome into one of the two daughter cells following cell division. It has been proposed that an active mechanism is used to ensure the health of one cell at the expense of the other (Fuentealba et al. 2008; Rujano et al. 2006). c There has been much discussion as to why asymmetric centrosome activity might be needed in stem cells; proper spindle alignment and asymmetric

progeny cells during budding yeast division. However, cortical ER inheritance is subject to an additional layer of regulation: under conditions of ER stress, both transport of cortical ER into the bud and cytokinesis are inhibited by a mechanism that involves the MAP kinase Slt2. Thus, inhibition of cortical ER partitioning during ER stress is a mechanism that prevents the detrimental inheritance of functionally compromised ER by the daughter cell, at the cost of selective retention in the mother. Whether a similar program of functional partitioning of the ER occurs in dividing animal cells is unknown but is an intriguing possibility worth pursuing.

Based on these examples from yeast in which mechanisms ensure that only highly functional mitochondria and ER are inherited by the more proliferative daughter cell, we would like to hypothesize that stem cells, or cells with high proliferation potential, need to maintain b

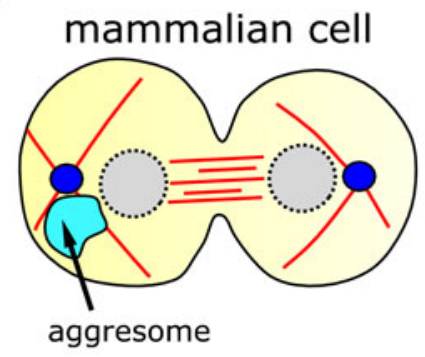

Post mitotic NB

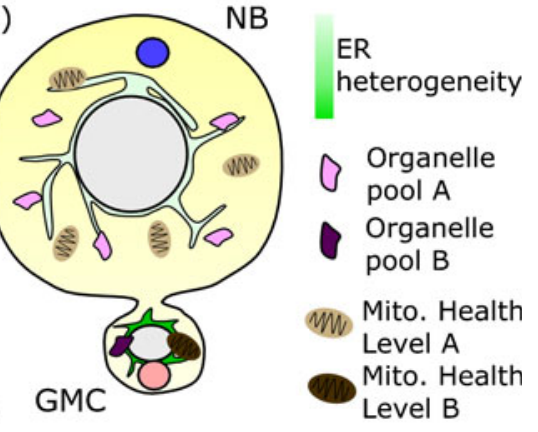

division are the most common reasons given. Here, we would like to add to the discussion by speculating that another purpose for maintaining a more active MTOC in interphase is the segregation of different organelle pools. Although evidence does not exist for such a mechanism in Drosophila NBs, other systems suggest that this type of regulation is plausible in stem cells. We hypothesize that the stem cell (NB) and differentiated cell (GMC) need to inherit a different subset of ER, mitochondria, other organelles, and mRNA (not depicted) to properly perform their respective functions

organelles with the highest possible fitness. We would also like to suggest that the presence of one large MTOC and one small MTOC could serve as a mechanism for segregating two populations of organelles, possibly the healthy and damaged (Fig. 4c). Is this truly a plausible hypothesis, and, if so, how might this occur? Similar to yeast, the ER in animal cells also associates extensively with the cytoskeleton, although in this case the associations are predominantly with MTs as opposed to actin (Friedman and Voeltz 2011; Griffing 2010). Accordingly, the ER associates with the mitotic spindle in dividing animal cells. More importantly, in all animal cell types examined, there is significant accumulation of the ER around the spindle poles (Bobinnec et al. 2003; Lu et al. 2009; Poteryaev et al. 2005; Puhka et al. 2007; Smyth et al. 2012; Terasaki 2000; Waterman-Storer et al. 1993). Thus, the ER is situated in close proximity to the centrosomes, and it is conceivable that in asymmetrically 
dividing stem cells, such as Drosophila NBs, the differentially regulated centrosomes may direct differential partitioning of ER to the progeny cells (Fig. 4c). A critical component of such a mechanism is the factors that link ER with the spindle poles and thus couple the organelle with the MTOC. A significant challenge for the field, therefore, is to identify the factors that couple the ER to MTs and investigate whether these factors can regulate biased ER segregation in asymmetrically dividing cells. Likewise, mitochondria use both plus-end and minus-end directed MT motors to traverse cells (Frederick and Shaw 2007). It is conceivable that mitochondria could also use MTOC asymmetry to segregate into a healthy pool and a damaged pool that are differentially inherited by the two daughter cells (Fig. 4c). Again, identifying the specific mechanisms and molecules (not just molecular motors) that partition mitochondria during cell division would be of great interest.

\section{Aggresomes and DNA circles}

As mentioned above, one rationale for asymmetrically segregating organelles is to prevent passage of accumulated damage to one daughter cell for the greater good of the tissue and organism. In this model of cellular altruism, damage is segregated to one sacrificial daughter, leaving one pristine daughter with improved fitness potential. Normally, damaged proteins are targeted for degradation via the proteasome, but misfolded proteins can overwhelm the quality control machinery, resulting in aggregate formation. Evidence from multiple systems shows that these aggregates are often inherited by one daughter cell in a centrosome-dependent manner.

Misfolded amyloid-like aggregates, called aggresomes, were characterized in human embryonic kidney cells as detergent-insoluble depots of multi-ubiquitinated proteins (Johnston et al. 1998). These aggresomes are encapsulated by intermediate filaments and, importantly, localize to centrosomes (Johnston et al. 1998) in a dynein-dependent manner (Johnston et al. 2002). Because aggresome formation has been linked to a number of severe human pathologies, including cystic fibrosis (Johnston et al. 1998) and Huntingtin's disease (Scherzinger et al. 1997), the mode of aggresome inheritance has been the topic of a number of studies. Intriguingly, asymmetric inheritance of aggresomes occurs during both symmetric and asymmetric mitotic divisions. In symmetrically dividing mammalian cells and in early Drosophila embryos, aggresomes accumulate around a single centrosome during interphase and are partitioned to one daughter cell following mitosis (Fig. 4b, Fuentealba et al. 2008; Rujano et al. 2006). Moreover, expansion of a single clone of Cos-7 cells shows a gradation of aggresome localization among sibling cells (Fuentealba et al. 2008), suggesting a possible link between aggresome localization and centrosome age. Aggresomes might also be partitioned in asymmetrically dividing stem cells. Studies suggest aggresomes may be excluded from putative intestinal stem cells, but appear to be enriched in differentiated crypt cells (Rujano et al. 2006). Although suggestive, these studies should be revisited using the Lgr5 intestinal stem cell marker (Barker et al. 2007). In contrast, aggresomes are retained in Drosophila embryonic neural stem cells (NBs), seemingly using the NB-destined centrosome as a mechanism of segregation (Rujano et al. 2006). These studies suggest preferential association with either mother or daughter centrosomes may mediate segregation of aggresomes. At this point, it is unclear if this association between aggresome asymmetry and centrosome asymmetry is required for cell health, but this model is worthy of investigation in the wellcharacterized Drosophila mGSCs and larval NBs. Would it be more advantageous for the organism to sequester aggresomes into the stem cell? This might not be unreasonable because the differentiated cells are critical for tissue function, whereas the stem cells might only be used as cell factories. It might be advantageous to encase all damaged material into a stem cell that eventually undergoes cell death during adulthood, such as the NB (Ito and Hotta 1992), or that can be replaced by a de-differentiation mechanism, such as the mGSCs (Brawley and Matunis 2004). The other possibility is that stem cells need to be cleared of all damaged protein; therefore, a mechanism that sequesters aggresomes to the differentiated daughter might be employed. It will be interesting to investigate a possible role for the interphase MTOC in stem cells as it relates to attracting or repelling aggresomes.

One well-known example of cellular altruism is the asymmetric retention of extrachromosomal rDNA circles (ERCs) in the mother cells of budding yeast. ERCs are DNA circles that arise from homologous recombination within an array of rDNA repeats. Early studies showed ERCs, which lack centromeres, 
preferentially segregate to the mother cell; moreover, ERC load is negatively correlated with yeast lifespan (Sinclair and Guarente 1997). The mechanism of ERC retention in yeast mother cells has been controversial (Khmelinskii et al. 2010; Shcheprova et al. 2008). Currently, the model that best reconciles data on ERC segregation is a passive diffusion mechanism where the narrow physical space that defines the bud neck, combined with the rapid yeast cell cycle, essentially blocks the passage of the deleterious ERC plasmids into the bud (Gehlen et al. 2011; Kennedy and McCormick 2011). Although no evidence suggests segregation of ERCs depends on SPBs and MTs, more work is required to directly test this possible model. Detailed discussion of this topic is beyond the scope of this review, but it does serve as a great example of sequestering damaged or harmful material into one cell for the greater good and survival of others.

\section{Endosomes}

Another example of centrosomes directing organelle positioning was shown in non-mitotic primary neuronal cultures, where centrosomes break the symmetry of cells by clustering organelles, such as Golgi and endosomes, at a site that will define the location of the first neurite extension (de Anda et al. 2005). Furthermore, recent work indicates endosome activity is directly regulated by centrosomes (Hehnly et al. 2012). Studies in mammalian cells indicate the endosome protein Rab11 associates with the mother centriole appendages, which regulate its activity (Hehnly et al. 2012). Importantly, these studies support the broad idea that asymmetric organelle localization, and unique biochemical activities, can be attributed to differences between mother and daughter centrosomes.

Asymmetric inheritance of Rab11 activity has also been described in Drosophila sensory organ precursor (SOP) cells. SOP cells are precursors of the peripheral nervous system that divide asymmetrically to generate either the neuronal pIIb cell, or the non-neuronal pIIa cell. Importantly, preferential association of Rab11 with one centrosome in the dividing SOP cell helps determine neuronal fate decisions by increasing Delta activity in pIIb through upregulating endocytosis (Emery et al. 2005). This is a great example of how centrosomes influence cell fate. Is Rab11 activity upregulated by the mother centrosome in SOP cells, as was found in mammalian cells (Hehnly et al. 2012)?
Although the alignment of centrosomes along the apical-basal polarity axis depends on both partner of inscuteable (Pins) and a network of planar cell polarity genes, it is still unknown if SOP cells preferentially segregate their mother and daughter centrosomes to the pIIa and pIIb progeny (Bellaiche et al. 2004; Gomes et al. 2009). It may also be informative to investigate Rab11 localization in cycling NBs and to examine rab11 mutants for NB amplification. Although dominant-negative Rab11 transgenes do not alter NB polarity (Halbsgut et al. 2011), it remains unclear if Rab11 is required for NB proliferation or GMC neuronal differentiation. Such studies are needed to probe whether centrosome-mediated regulation of endocytosis is a general mechanism in stem cell fate specification.

\section{Relaying the message}

In addition to sorting organelles, cells must regulate another critical cellular element during symmetric and asymmetric divisions-messenger RNA (mRNA). Asymmetric mRNA localization is a critical paradigm in regulating the temporal and spatial activities of a large number of proteins (Gavis et al. 2007). For example, a common mechanism used for partitioning cell fate determinants is the differential localization and translational regulation of specific mRNAs (Medioni et al. 2012).

One mechanism of localizing specific transcripts within the cells is by a physical interaction between mRNA and the ER, as seen in the yeast bud (Jansen et al. 1996). Another mechanism implicates centrosomes in the segregation of mRNAs in a variety of systems including Spisula (Alliegro et al. 2006), Xenopus (Blower et al. 2007), and Drosophila (Lecuyer et al. 2007). The germ plasm of the early Drosophila embryo serves as a useful example for the asymmetric association of mRNAs with centrosomes. Germ plasm is a specialized cytoplasm that contains the mRNAs and proteins that are both necessary and sufficient for germline development (Strome and Lehmann 2007). Recent live-cell-imaging studies of nanos mRNA (nos) indicate germ plasm mRNAs undergo directed dyneindependent transport to centrosomes to ensure their passage into primordial germ cells (Lerit and Gavis 2011). In cases where nuclei divide orthogonal to the posterior pole, nos mRNA and other germ plasm components only associate with the most proximal MTOC. Ectopically localizing germ plasm along the syncytial 
embryo cortex demonstrated that all centrosomes are equally capable of recruiting germ plasm in Drosophila (Lerit and Gavis 2011). This system shows careful timing of MTOC migration in the early embryo guides critical cell-fate decisions.

Could stem cells use a similar mechanism to asymmetrically segregate mRNAs? The presence of one active interphase MTOC might serve as an ideal mechanism to sequester transcripts destined for the stem cell. High-throughput analysis revealed that over 3,500 transcripts are differentially expressed in NBs and GMCs (Berger et al. 2012). Do any of these mRNAs associate specifically with mother or daughter centrosomes? We predict that biochemical isolation of specific centrosomes, perhaps coupled with the detection of mother or daughter centrosome-specific proteins, followed by RNA-seq, will uncover a large pool of transcripts that guide cell-fate decisions. Modulating MTOC size and position are likely critical to ensure passage of such determinants in a number of stem cell systems.

"Some (MTOCs) are born great, some (eventually) achieve greatness"

To better understand the asymmetry, or delay, in interphase MTOC activity and its functional significance, we must investigate the mechanisms that control MT organization, which encompasses MT nucleation, MT anchoring, and MT severing/release at the centrosome. Intuitively, these mechanisms must be differentially modulated on the mother and daughter centrosomes by varying the activity of positive or negative regulators. In the following section, we discuss three possible mechanisms of establishing asymmetry in MTOC activity.

\section{Centriole ultrastructure and distinct protein composition (intrinsic to the centrosome)}

We highlighted in Fig. 1 the normal process of centriole duplication where the $\mathrm{M}$ and $\mathrm{D}$ centrioles in S-phase give rise to the GD1 and GD2 centrioles, respectively. Importantly, the more mature $\mathrm{M}$ centriole assembles distinctive structures at its distal ends referred to as distal and sub-distal appendages. These appendages have long been described using serial section EM in mammalian cells (Vorobjev and Chentsov 1980) and many species (Carvalho-Santos et al. 2011). To date, several proteins have been reported to exclusively localize to these appendages, such as $\varepsilon$-Tubulin, Centriolin, Ninein, EB1, Cep170, Kif24, Cep164, Odf2, and the DAP proteins (Brito et al. 2012; Tanos et al. 2013). It is, therefore, simple to conceptualize a linear relationship between M-centriole age $\rightarrow$ ultrastructural differences (appendages) $\rightarrow$ specialized protein composition $\rightarrow$ more active MTOC. This mechanism could be used by mammalian systems such as the RGCs, but a different mechanism must be employed by species that do not have distinct ultrastructural differences between the $\mathrm{M}$ and $\mathrm{D}$ centrioles, like Drosophila (Callaini and Riparbelli 1990). It is, therefore, not surprising that none of the aforementioned appendage proteins have been identified as Drosophila M centriole proteins. However, Drosophila cells can still distinguish the $\mathrm{M}$ and $\mathrm{D}$ centriole using daughtercentriole specific proteins (Januschke et al. 2011). Several of these daughter specific proteins, such as Centrobin, Neural4, and Cep120, have been identified. Therefore, different species use different mechanisms to distinguish the $\mathrm{M}$ and $\mathrm{D}$ centrioles. The above model should then be modified to be more general, stating that $\mathrm{M}$ or $\mathrm{D}$ specific centriole proteins $\rightarrow$ a more active MTOC (Fig. 3).

We must keep in mind that these protein differences are between $\mathrm{M}$ and $\mathrm{D}$ centrioles of a single centrosome, not necessarily between the mother and daughter centrosomes. How might the centriole differences translate into centrosome differences as they relate to MTOC activity? The relationships between the four centrioles, the two centrosomes, and MTOC activity in late interphase and mitosis are often not discussed due to their complexity, but the details are important to understand. We will use the M, D, GD1, and GD2 nomenclature presented above. The single G1 centrosome contains the $\mathrm{M}$ centriole with mother protein composition and the $\mathrm{D}$ centriole with daughter protein composition. To properly investigate the role of the $\mathrm{M}$ and D specific proteins in asymmetric MTOC activity, we must consider the following: (1) when does the D centriole lose daughter centriole proteins and gain mother centriole proteins; (2) when exactly are the GD1 and GD2 centrioles nucleated; (3) when do the GD1 and GD2 centrioles acquire daughter centriole proteins; (4) can GD1 and GD2 differ in protein composition at the same point in the cell cycle; and (5) do centrioles act autonomously, or can the protein composition of one centriole influence its engaged partner? It will be challenging to follow all of these events, 
but it is clear that live cell imaging using specific $\mathrm{M}$ and D markers, combined with indicators of MTOC activity and mutant analysis, are all needed to truly understand the mechanisms that generate asymmetry. Added to this complexity is the fact that MTOC activity ( $\gamma$-tubulin recruitment) proceeds through at least three overlapping pathways ( $\operatorname{Spd} 2$, Pericentrin, and Centrosomin; Haren et al. 2009), and regulation of these pathways might differ in interphase and mitosis. Thoroughly understanding centrosome maturation, and how it is uniquely controlled on mother versus daughter centrosomes, in stem cells that divide and segregate organelles asymmetrically will require a concerted effort by many laboratories.

Local environment (extrinsic to the centrosome) Another possible mechanism of distinguishing the two centrosomes is by varying the local environment. In this model, a fixed signal with limited range within the cell would modulate MTOC activity. This mechanism seems to be employed by some cells. For example, the SPB inheritance pattern in budding yeast can be randomized by depolymerizing microtubules, allowing the SPBs to move freely within the nuclear membrane. Upon drug washout, the SPB nearest the bud neck gains MTOC activity and is inherited by the bud (Pereira et al. 2001). This demonstrates that MTOC capacity might be dictated by the SPB's proximity to the bud neck, not by an inherent difference in motherdaughter SPB identity. However, the timing of drug washout in this experiment should be considered in light of the 10-min delay between the mother and daughter SPB maturation in interphase (Shaw et al. 1997). It is possible if the washout was performed within this 10-min time window that only the mother SPB will nucleate MTs, get pulled toward the bud neck, and become inherited properly by the bud.

In Drosophila mGSCs, an intracellular signaling mechanism that is extrinsic to the centrosome seems to be employed to distinguish differential MTOC activity (Inaba et al. 2010; Yamashita et al. 2003), although MT depolymerization or in vitro culture experiments have not been performed to test this mechanism. In Drosophila NBs, a similar signaling mechanism seems to influence MTOC activity. Loss of the apical determinant gene, pins, leads to loss of apical daughter centrosome activity, resulting in two inactive centrosomes (Januschke et al. 2013). However, there seems to be an important feedback loop between the apical centrosome and the apical cortex because localization of apical determinants themselves relies on MTOC activity (Januschke and Gonzalez 2010). Such a feedback mechanism might be critical in cells that do not have a stem cell niche. It will be important to further investigate the communication between the cortex and the active MTOC in interphase. It might be interesting to determine the effects of repositioning the local environment, for example, by relocating the apical domain to the basal side of the NB to test the effect on MTOC activity.

It is noteworthy that the two centrioles (or SPBs) start out adjacent to one another in G1 and, thus, are initially subject to the same local environment. This indicates that an extrinsic signal does not function in isolation and can only act on centrioles with specific $\mathrm{M}$ or D identity to induce asymmetric MTOC activity.

Limited material A relatively unexplored area of possible regulation is the presence of limiting amounts of an MTOC regulator. It is known that global changes in PCM and centriole levels can influence their size and number (Goehring and Hyman 2012), but is this mechanism employed by stem cells to generate MTOC asymmetry? In such a model, a finite amount of a positive regulator would initially be equally distributed between the mother and daughter centrosome. However, the on-off cycling of such a regulator could briefly favor one of the two centrosomes, which would slightly increase the MTOC capacity of that centrosome. This, in turn, might increase its sequestration capacity, possibly attracting the remaining molecules of this regulator. In this fashion, one centrosome will self-amplify, whereas the other centrosome will remain inactive. This positive feedback mechanism is supported by recent computational modeling that suggests a slight initial imbalance in centrosome activity can lead to a great imbalance in sequestered cargo (Chen et al. 2012). This model is not without its caveats. Most importantly, this model predicts an equal probability that one centrosome will become the active MTOC, and this is certainly not the case in stem cells. However, in combination with mechanisms that recognize $\mathrm{M}-\mathrm{D}$ centriole specific proteins or structural differences, this could augment MTOC asymmetry. Specifically, a very early (G1) protein difference (Cep164, for example) between $\mathrm{M}$ and $\mathrm{D}$ could serve as the tipping point that favors the recruitment of a regulator to the $\mathrm{M}$ centriole, and thus the 
mother centrosome. This would occur immediately after mitotic exit and, most importantly, prior to the recruitment of the same protein to the $\mathrm{D}$ centriole as it matures much later in the cell cycle. At this point, the positive feedback mechanism is already engaged to maintain activity at the mother centrosome.

In summary, each of these models offers a plausible mechanism of establishing MTOC asymmetry. However, it is likely that cells use a combination of these mechanisms (and others) to different degrees depending on cell type. It is our opinion that in order to fully understand this process, future research must move away from the "hammer" experiments that completely disrupt centrosomes (as we have done, Rusan and Peifer 2007), away from disassembling the entire MT network, away from eliminating any and all cell polarity, and away from strictly analyzing protein nulls. We must instead work toward a true molecular understanding of PCM recruitment or centrosome maturation. The literature does provide examples of proteins that are implicated in establishing MTOC asymmetry, such as Kar9 (Liakopoulos et al. 2003), Polo (Rusan and Peifer 2007), Cnn (Conduit and Raff 2010), BRCA1 (Tarapore et al. 2012), Szy20 (Song et al. 2008), and Ninein (Wang et al. 2009). We will not discuss the details of these studies here, but we will present the findings of a recently identified regulator of MTOC asymmetry-Centrobin (Cnb).

MTOC asymmetry in NBs - the positive and the negative

It has been proposed that the striking MTOC asymmetry observed in interphase NBs is important for orienting the invariant mitotic spindle axis that drives asymmetric division of these cells (Rebollo et al. 2007; Rusan and Peifer 2007). Recent work has begun to explore the mechanisms that regulate asymmetric centrosome maturation. Mutant analysis and ectopic localization studies show that the daughter-centriole protein $\mathrm{Cnb}$ is a critical determinant of centrosome maturation (Januschke et al. 2013). Loss of cnb eliminates the active interphase centrosome and randomizes the mother-daughter centrosome inheritance pattern, while targeting Cnb to all centrioles with a PACT tag generates two active interphase centrosomes (Januschke et al. 2013). In both cases, mitotic centrosome maturation and asymmetric cell divisions appear to occur normally. These data indicate that $\mathrm{Cnb}$ is an important positive regulator used to establish MTOC asymmetry and to target the daughter centrosome to the stem cell. However, the physiological consequence of disrupting the centrosome inheritance pattern is still unknown. It remains formally possible that randomization of centrosome inheritance may result in subtle or more long-term phenotypes that have thus far eluded researchers.

The above study shows a positive regulator specifically targeted to the daughter centrosome, but it might also be the case that a negative regulator could be enriched on the mother centrosome. Analysis of PCM dynamics, for example, indicates the incorporation rate of $\mathrm{Cnn}$ is faster at the daughter, active centrosome (Conduit and Raff 2010). However, it is still unclear how mother centrosomes slow down Cnn turnover (Conduit and Raff 2010). It will be interesting to determine if $\mathrm{Cnb}$ directly increases $\mathrm{Cnn}$ turnover on the daughter centrosome, and to possibly identify factors (negative regulators) that stabilize $\mathrm{Cnn}$ at the mother centrosome.

\section{Stem cells-WANTED ALIVE}

The study mentioned above has only scratched the surface of our understanding of centrosome maturation and inheritance asymmetry. To explore this model further, we must employ all the tools at our disposal, specifically tools to monitor live cells. Techniques such as fluorescence recovery after photobleaching, photoconversion, laser ablation, and detailed fluorescence quantification throughout the cell cycle must be the standard. For example, it was only live cell imaging that uncovered the delay in centrosome maturation in interphase SPBs (Shaw et al. 1997) and NB centrosomes (Rebollo et al. 2007; Rusan and Peifer 2007). Extensive live analysis of the mGSCs and mouse RGCs is required to solve the loose ends left by fixed analyses of these cells.

One unexplored area that demands live cell imaging to test the importance of centrosome asymmetry and inheritance is the transition between symmetric and asymmetric stem cell divisions. Stem cells undergo symmetric cell division during periods of stem cell expansion, a process that is important for tissue repair and regeneration. Recent live cell imaging of the Drosophila testes, for example, indicates symmetric 
mGSC division occurs with some frequency in normal tissue (Sheng and Matunis 2011). These observations argue that centrosome age alone is insufficient to dictate cell fate decisions. Another unexplored system is the period of stem cell expansion during early CNS development in Drosophila. During this period, first-instar larvae significantly increase the number of central brain NBs, the same cells that will later display stereotypical centrosome inheritance patterns and differential MTOC activity. Observations of these wild-type cells will shed light on the regulation and importance of asymmetric centrosome activity on cell fate. For example, does $\mathrm{Cnb}$ or other maturation factors localize evenly between the two centrosomes during early NB symmetric divisions, and are they subsequently restricted to one centrosome in the asymmetric NB divisions of second- and third-instar larvae? And how are organelles partitioned in such divisions?

\section{Concluding remarks}

Data from disparate model organisms suggest that centrosomes are important mediators of organelle and mRNA segregation. Unequal partitioning of these specific organelles and transcripts, especially during asymmetric stem cell divisions, is likely critical for cellular health and fate. Interestingly, centrosome age has been linked to asymmetric segregation of organelles and mRNAs in non-stem cell systems. Moving forward, it is of utmost importance to determine if the nonrandom centrosome segregation in asymmetrically dividing stem cells contributes to the partitioning of healthy versus diseased (or otherwise modified) organelles. To date, no real functional consequence of randomizing centrosome inheritance patterns in stem cells has been identified, but a closer look at other cellular components might reveal the significance of this invariant segregation of mother and daughter centrosomes. We believe that the Drosophila neural stem cells are the most suitable system to investigate the process of asymmetric organelle inheritance and will continue to serve as a powerful system to guide experimentation in mouse and human cells.

Acknowledgments We would like to thank Brian Galletta, Carey Fagerstrom, and the anonymous reviewers for their insightful comments. We apologize to those authors whose work we were not able to cite due to space constrains.

\section{References}

Alliegro MC, Alliegro MA, Palazzo RE (2006) Centrosomeassociated RNA in surf clam oocytes. [Research Support, N.I.H., Extramural]. Proc Natl Acad Sci U S A 103(24): 9034-9038

Anderson CT, Stearns T (2009) Centriole age underlies asynchronous primary cilium growth in mammalian cells. [Research Support, N.I.H., Extramural. Research Support, U.S. Gov't, Non-P.H.S.]. Curr Biol 19(17):1498-1502

Barker N, van Es JH, Kuipers J, Kujala P, van den Born M, Cozijnsen $M$ et al (2007) Identification of stem cells in small intestine and colon by marker gene Lgr5. Nature 449(7165):1003-1007

Bellaiche Y, Beaudoin-Massiani O, Stuttem I, Schweisguth F (2004) The planar cell polarity protein Strabismus promotes Pins anterior localization during asymmetric division of sensory organ precursor cells in Drosophila. [Research Support, Non-U.S. Gov't]. Development 131(2):469-478

Berger C, Harzer H, Burkard TR, Steinmann J, van der Horst S, Laurenson AS et al (2012) FACS purification and transcriptome analysis of Drosophila neural stem cells reveals a role for Klumpfuss in self-renewal. [Research Support, Non-U.S. Gov't]. Cell Reports 2(2):407-418

Blower MD, Feric E, Weis K, Heald R (2007) Genome-wide analysis demonstrates conserved localization of messenger RNAs to mitotic microtubules. J Cell Biol 179(7):13651373

Bobinnec Y, Marcaillou C, Morin X, Debec A (2003) Dynamics of the endoplasmic reticulum during early development of Drosophila melanogaster. Cell Motil Cytoskeleton 54(3):217-225

Brawley C, Matunis E (2004) Regeneration of male germline stem cells by spermatogonial dedifferentiation in vivo. Science 304(5675):1331-1334

Brito DA, Gouveia SM, Bettencourt-Dias M (2012) Deconstructing the centriole: structure and number control. [Research Support, Non-U.S. Gov't Review]. Curr Opin Cell Biol 24(1):4-13

Callaini G, Riparbelli MG (1990) Centriole and centrosome cycle in the early Drosophila embryo. [Research Support, Non-U.S. Gov't]. J Cell Sci 97(Pt 3):539-543

Carvalho-Santos Z, Azimzadeh J, Pereira-Leal JB, BettencourtDias M (2011) Evolution: tracing the origins of centrioles, cilia, and flagella. [Research Support, Non-U.S. Gov't Review]. J Cell Biol 194(2):165-175

Chen J, Lippincott-Schwartz J, Liu J (2012) Intracellular spatial localization regulated by the microtubule network. PLoS One 7(4):e34919

Conduit PT, Raff JW (2010) Cnn dynamics drive centrosome size asymmetry to ensure daughter centriole retention in Drosophila neuroblasts. Curr Biol 20(24): 2187-2192 
Conduit PT, Brunk K, Dobbelaere J, Dix CI, Lucas EP, Raff JW (2010) Centrioles regulate centrosome size by controlling the rate of $\mathrm{Cnn}$ incorporation into the PCM. Curr Biol 20(24):2178-2186

de Anda FC, Pollarolo G, Da Silva JS, Camoletto PG, Feiguin F, Dotti CG (2005) Centrosome localization determines neuronal polarity. [Research Support, Non-U.S. Gov't]. Nature 436(7051):704-708

Du Y, Ferro-Novick S, Novick P (2004) Dynamics and inheritance of the endoplasmic reticulum. J Cell Sci 117(Pt 14):2871-2878

Emery G, Hutterer A, Berdnik D, Mayer B, Wirtz-Peitz F, Gaitan MG et al (2005) Asymmetric Rab 11 endosomes regulate delta recycling and specify cell fate in the Drosophila nervous system. [Research Support, Non-U.S. Gov't]. Cell 122(5):763-773

Fehrenbacher KL, Yang H-C, Gay AC, Huckaba TM, Pon LA (2004) Live cell imaging of mitochondrial movement along actin cables in budding yeast. Curr Biol 14(22):1996-2004

Frederick RL, Shaw JM (2007) Moving mitochondria: establishing distribution of an essential organelle. Traffic 8(12):1668-1675

Friedman JR, Voeltz GK (2011) The ER in 3D: a multifunctional dynamic membrane network. Trends Cell Biol 21(12):709-717

$\mathrm{Fu}$ J, Glover DM (2012) Structured illumination of the interface between centriole and peri-centriolar material. Open Biology 2(8):120104

Fuentealba LC, Eivers E, Geissert D, Taelman V, De Robertis EM (2008) Asymmetric mitosis: unequal segregation of proteins destined for degradation. [Research Support, N.I.H., Extramural. Research. Support, Non-U.S. Gov't]. Proc Natl Acad Sci U S A 105(22)):7732-7737

Fuller MT (1993) Spermatogenesis. In: Bate M, Martinez Arias A (eds) The development of Drosophila melanogaster. CSHL, Cold Spring Harbor, pp 71-147

Gavis ER, Singer RH, Huttelmaier S (2007) Localized translation through messenger RNA localization. In: Hershey JWB, Mathews MB, Sonenberg N (eds) Translational control in biology and medicine, vol 48. Cold Spring Harbor, New York, pp 687-717

Gehlen LR, Nagai S, Shimada K, Meister P, Taddei A, Gasser SM (2011) Nuclear geometry and rapid mitosis ensure asymmetric episome segregation in yeast. [Research Support, Non-U.S. Gov't]. Curr Biol 21(1):25-33

Goehring NW, Hyman AA (2012) Organelle growth control through limiting pools of cytoplasmic components. Curr Biol 22(9):R330-339

Gomes JE, Corado M, Schweisguth F (2009) Van Gogh and Frizzled act redundantly in the Drosophila sensory organ precursor cell to orient its asymmetric division. [Research Support, Non-U.S. Gov't. Research Support, U.S. Gov't, Non-P.H.S.]. PLoS One 4(2):e4485

Griffing LR (2010) Networking in the endoplasmic reticulum. Biochem Soc Trans 38(3):747-753

Halbsgut N, Linnemannstons K, Zimmermann LI, Wodarz A (2011) Apical-basal polarity in Drosophila neuroblasts is independent of vesicular trafficking. [Research Support, Non-U.S. Gov't]. Mol Biol Cell 22(22):4373-4379

Haren L, Stearns T, Luders J (2009) Plk1-dependent recruitment of gamma-tubulin complexes to mitotic centrosomes involves multiple PCM components. [Research Support, N.I.H., Extramural. Research Support, Non-U.S. Gov't]. PLoS One 4(6):e5976

Hehnly H, Chen CT, Powers CM, Liu HL, Doxsey S (2012) The centrosome regulates the Rab11-dependent recycling endosome pathway at appendages of the mother centriole. [Research Support, N.I.H., Extramural]. Curr Biol 22(20):1944-1950

Henderson KA, Gottschling DE (2008) A mother's sacrifice: what is she keeping for herself? [Review]. Curr Opin Cell Biol 20(6):723-728

Homem CC, Knoblich JA (2012) Drosophila neuroblasts: a model for stem cell biology. Development 139(23):42974310

Hotz M, Leisner C, Chen D, Manatschal C, Wegleiter T, Ouellet $\mathrm{J}$ et al (2012) Spindle pole bodies exploit the mitotic exit network in metaphase to drive their age-dependent segregation. [Research Support, N.I.H., Extramural Research Support, Non-U.S. Gov't]. Cell 148(5):958-972

Inaba M, Yuan H, Salzmann V, Fuller MT, Yamashita YM (2010) E-cadherin is required for centrosome and spindle orientation in Drosophila male germline stem cells. [Research Support, N.I.H., Extramural Research Support, Non-U.S. Gov't]. PLoS One 5(8):e12473

Ito K, Hotta Y (1992) Proliferation pattern of postembryonic neuroblasts in the brain of Drosophila melanogaster. Dev Biol 149(1):134-148

Izumi H, Kaneko Y (2012) Evidence of asymmetric cell division and centrosome inheritance in human neuroblastoma cells. [Research Support, Non-U.S. Gov't]. Proc Natl Acad Sci U S A 109(44):18048-18053

Jansen RP, Dowzer C, Michaelis C, Galova M, Nasmyth K (1996) Mother cell-specific HO expression in budding yeast depends on the unconventional myosin myo4p and other cytoplasmic proteins. [Research Support, Non-U.S. Gov't]. Cell 84(5):687-697

Januschke J, Gonzalez C (2010) The interphase microtubule aster is a determinant of asymmetric division orientation in Drosophila neuroblasts. J Cell Biol 188(5):693706

Januschke J, Llamazares S, Reina J, Gonzalez C (2011) Drosophila neuroblasts retain the daughter centrosome. Nat Commun 2:243

Januschke J, Reina J, Llamazares S, Bertran T, Rossi F, Roig J, et al. (2013) Centrobin controls mother-daughter centriole asymmetry in Drosophila neuroblasts. Nat Cell Biol 15:241-248

Jiang M, Stanke J, Lahti JM (2011) The connections between neural crest development and neuroblastoma. [Research Support, N.I.H., Extramural Research Support, Non-U.S. Gov't Review]. Curr Top Dev Biol 94:77-127

Johnston JA, Ward CL, Kopito RR (1998) Aggresomes: a cellular response to misfolded proteins. [Research Support, Non-U.S. Gov't Research Support, U.S. Gov't, P.H.S.]. J Cell Biol 143(7):1883-1898

Johnston JA, Illing ME, Kopito RR (2002) Cytoplasmic dynein/ dynactin mediates the assembly of aggresomes. [Research Support, U.S. Gov't, P.H.S.]. Cell Motil Cytoskeleton 53(1):26-38

Kennedy BK, McCormick MA (2011) Asymmetric segregation: the shape of things to come? Curr Biol 21(4):R149-151 
Khmelinskii A, Keller PJ, Lorenz H, Schiebel E, Knop M (2010) Segregation of yeast nuclear pores. Nature 466(7305):E1

Khodjakov A, Rieder CL (1999) The sudden recruitment of gamma-tubulin to the centrosome at the onset of mitosis and its dynamic exchange throughout the cell cycle, do not require microtubules. [Research Support, U.S. Gov't, P.H.S.]. J Cell Biol 146(3):585-596

Lawo S, Hasegan M, Gupta GD, Pelletier L (2012) Subdiffraction imaging of centrosomes reveals higher-order organizational features of pericentriolar material. [Research Support, NonU.S. Gov't]. Nat Cell Biol 14(11):1148-1158

Lecuyer E, Yoshida H, Parthasarathy N, Alm C, Babak T, Cerovina $\mathrm{T}$ et al (2007) Global analysis of mRNA localization reveals a prominent role in organizing cellular architecture and function. [Research Support, Non-U.S. Gov't]. Cell 131(1):174-187

Lerit DA, Gavis ER (2011) Transport of germ plasm on astral microtubules directs germ cell development in Drosophila. Curr Biol 21(6):439-448

Lesage B, Gutierrez I, Marti E, Gonzalez C (2010) Neural stem cells: the need for a proper orientation. Curr Opin Genet Dev 20(4):438-442

Liakopoulos D, Kusch J, Grava S, Vogel J, Barral Y (2003) Asymmetric loading of Kar9 onto spindle poles and microtubules ensures proper spindle alignment. [Research Support, Non-U.S. Gov't]. Cell 112(4):561-574

Lowe M, Barr FA (2007) Inheritance and biogenesis of organelles in the secretory pathway. Nat Rev Mol Cell Biol 8(6):429-439. doi:10.1038/nrm2179

Lu L, Ladinsky MS, Kirchhausen T (2009) Cisternal organization of the endoplasmic reticulum during mitosis. Mol Biol Cell 20(15):3471-3480

Medioni C, Mowry K, Besse F (2012) Principles and roles of mRNA localization in animal development. Development 139(18):3263-3276

Mennella V, Keszthelyi B, McDonald KL, Chhun B, Kan F, Rogers GC et al (2012) Subdiffraction-resolution fluorescence microscopy reveals a domain of the centrosome critical for pericentriolar material organization. [Research Support, N.I.H., Extramural Research Support, Non-U.S. Gov't]. Nat Cell Biol 14(11):1159-1168

Morrison SJ, Spradling AC (2008) Stem cells and niches: mechanisms that promote stem cell maintenance throughout life. [Research Support, Non-U.S. Gov't Review]. Cell 132(4):598-611

Peraza-Reyes L, Crider DG, Pon LA (2010) Mitochondrial manoeuvres: latest insights and hypotheses on mitochondrial partitioning during mitosis in Saccharomyces cerevisiae. BioEssays 32(12):1040-1049

Pereira G, Tanaka TU, Nasmyth K, Schiebel E (2001) Modes of spindle pole body inheritance and segregation of the Bfa1p-Bub2p checkpoint protein complex. [Research Support, Non-U.S. Gov't]. EMBO J 20(22): 6359-6370

Poteryaev D, Squirrell JM, Campbell JM, White JG, Spang A (2005) Involvement of the actin cytoskeleton and homotypic membrane fusion in ER dynamics in Caenorhabditis elegans. Mol Biol Cell 16(5):2139-2153

Puhka M, Vihinen H, Joensuu M, Jokitalo E (2007) Endoplasmic reticulum remains continuous and undergoes sheet-to-tubule transformation during cell division in mammalian cells. J Cell Biol 179(5):895-909

Rebollo E, Sampaio P, Januschke J, Llamazares S, Varmark H, Gonzalez C (2007) Functionally unequal centrosomes drive spindle orientation in asymmetrically dividing Drosophila neural stem cells. Dev Cell 12(3):467-474

Rose MD, Miller RK (1998) Kar9p is a novel cortical protein required for cytoplasmic microtubule orientation in yeast. [Research Support, U.S. Gov't, P.H.S.]. J Cell Biol 140(2):377-390

Rujano MA, Bosveld F, Salomons FA, Dijk F, van Waarde MA, van der Want JJ (2006) Polarised asymmetric inheritance of accumulated protein damage in higher eukaryotes. [Research Support, Non-U.S. Gov't]. PLoS Biology 4(12):e417

Rusan NM, Peifer M (2007) A role for a novel centrosome cycle in asymmetric cell division. J Cell Biol 177(1):13-20

Scherzinger E, Lurz R, Turmaine M, Mangiarini L, Hollenbach B, Hasenbank R et al (1997) Huntingtin-encoded polyglutamine expansions form amyloid-like protein aggregates in vitro and in vivo. [Research Support, Non-U.S. Gov't]. Cell 90(3):549-558

Shaw SL, Yeh E, Maddox P, Salmon ED, Bloom K (1997) Astral microtubule dynamics in yeast: a microtubulebased searching mechanism for spindle orientation and nuclear migration into the bud. [Research Support, NonU.S. Gov't Research Support, U.S. Gov't, P.H.S.]. J Cell Biol 139(4):985-994

Shcheprova Z, Baldi S, Frei SB, Gonnet G, Barral Y (2008) A mechanism for asymmetric segregation of age during yeast budding. Nature 454(7205):728-734

Sheng XR, Matunis E (2011) Live imaging of the Drosophila spermatogonial stem cell niche reveals novel mechanisms regulating germline stem cell output. [Research Support, N.I.H., Extramural]. Development 138(16):3367-3376

Simon VR, Karmon SL, Pon LA (1997) Mitochondrial inheritance: cell cycle and actin cable dependence of polarized mitochondrial movements in Saccharomyces cerevisiae. Cell Motil Cytoskeleton 37(3):199-210

Sinclair DA, Guarente L (1997) Extrachromosomal rDNA circles - a cause of aging in yeast. [Research Support, NonU.S. Gov't Research Support, U.S. Gov't, P.H.S.]. Cell 91(7):1033-1042

Smyth JT, Beg AM, Wu S, Jr P, James W, Rusan NM (2012) Phosphoregulation of STIM1 leads to exclusion of the endoplasmic reticulum from the mitotic spindle. Curr Biol 22(16):1487-1493

Song MH, Aravind L, Muller-Reichert T, O'Connell KF (2008) The conserved protein SZY-20 opposes the Plk4-related kinase ZYG-1 to limit centrosome size. [Research Support, N.I.H., Extramural Research Support, N.I.H., Intramural]. Dev Cell 15(6):901-912

Strome S, Lehmann R (2007) Germ versus soma decisions: lessons from flies and worms. Science 316(5823): 392-393

Sunkel CE, Gomes R, Sampaio P, Perdigao J, Gonzalez C (1995) Gamma-tubulin is required for the structure and function of the microtubule organizing centre in 
Drosophila neuroblasts. [Research Support, Non-U.S. Gov't]. EMBO J 14(1):28-36

Tanos BE, Yang HJ, Soni R, Wang WJ, Macaluso FP, Asara JM et al (2013) Centriole distal appendages promote membrane docking, leading to cilia initiation. Genes Dev 27(2):163-168

Tarapore P, Hanashiro K, Fukasawa K (2012) Analysis of centrosome localization of BRCA1 and its activity in suppressing centrosomal aster formation. [Research Support, N.I.H., Extramural]. Cell Cycle 11(15):2931-2946

Terasaki M (2000) Dynamics of the endoplasmic reticulum and Golgi apparatus during early sea urchin development. Mol Biol Cell 11(3):897-914

Vallen EA, Scherson TY, Roberts T, van Zee K, Rose MD (1992) Asymmetric mitotic segregation of the yeast spindle pole body. [Research Support, Non-U.S. Gov't Research Support, U.S. Gov't, Non-P.H.S. Research Support, U.S. Gov't, P.H.S.]. Cell 69(3):505-515

Vorobjev IA, Chentsov YS (1980) The ultrastructure of centriole in mammalian tissue culture cells. [Comparative Study]. Cell Biol Int Rep 4(11):1037-1044

Wang X, Tsai JW, Imai JH, Lian WN, Vallee RB, Shi SH (2009) Asymmetric centrosome inheritance maintains neural progenitors in the neocortex. [Research Support, N.I.H., Extramural Research Support, Non-U.S. Gov't]. Nature 461(7266):947-955

Waterman-Storer CM, Sanger JW, Sanger JM (1993) Dynamics of organelles in the mitotic spindles of living cells: membrane and microtubule interactions. Cell Motil Cytoskeleton 26(1):19-39

Yamashita YM, Fuller MT (2008) Asymmetric centrosome behavior and the mechanisms of stem cell division. J Cell Biol 180(2):261-266

Yamashita YM, Jones DL, Fuller MT (2003) Orientation of asymmetric stem cell division by the APC tumor suppressor and centrosome. [Research Support, Non-U.S. Gov't Research Support, U.S. Gov't, P.H.S.]. Science 301(5639):1547-1550

Yamashita YM, Mahowald AP, Perlin JR, Fuller MT (2007) Asymmetric inheritance of mother versus daughter centrosome in stem cell division. [Research Support, Non-U.S. Gov't Research Support, U.S. Gov't, P.H.S.]. Science 315(5811):518-521

Yang H-C, Pon LA (2002) Actin cable dynamics in budding yeast. Proc Natl Acad Sci 99(2):751-756 